\title{
The Molecular Choreography of IRF4 and IRF8 with Immune System Partners
}

\author{
Harinder Singh, ${ }^{1,2,4}$ Elke Glasmacher, ${ }^{1,3}$ Abraham B. Chang, ${ }^{1}$ \\ AND BRYAN VANDER LUGT ${ }^{1}$ \\ ${ }^{1}$ Department of Discovery Immunology, Genentech Inc., South San Francisco, California 94080 \\ ${ }^{4}$ Correspondence: Harinder.Singh@cchmc.org
}

\begin{abstract}
The transcription factors IRF4 and IRF8 represent immune-specific members of the interferon regulatory family. They play major roles in controlling the development and functioning of innate and adaptive cells. Genes encoding these factors appear to have been coopted by the immune system via gene duplication and divergence of regulatory and protein coding sequences to enable the acquisition of unique molecular properties and functions. Unlike other members of the IRF family, IRF4 and IRF8 do not activate transcription of Type 1 interferon genes or positively regulate interferon-induced gene expression. Instead, they bind to unusual composite Ets-IRF or AP-1-IRF elements with specific Ets or AP-1 family transcription factors, respectively, and regulate the expression of diverse sets of immune response genes in innate as well as adaptive cells.
\end{abstract}

The molecular cloning of interferon regulatory factor 8 (IRF8) as interferon consensus sequence-binding protein (ICSBP) led to the realization that it bound interferon response sequence elements (ISRE), albeit with low affinity, and antagonized ISRE-mediated gene activation (Driggers et al. 1990). A breakthrough came with the characterization of IRF4 (Pip/ICSAT/LSIRF), which revealed not only its structural relatedness to IRF8 but also its unusual property of cooperatively assembling with the Ets family factor PU.1 on Ets-IRF composite elements (EICEs) (Eisenbeis et al. 1995). IRF8 shared the same distinctive molecular property and this led to the concept that key functions of IRF4 and IRF8 in B lineage and myeloid cells could be executed via complexes with PU.1 assembled on EICE motifs. IRF4 and IRF8 function equivalently in controlling pre-B-cell differentiation ( $\mathrm{Lu}$ et al. 2003). IRF4, on the other hand, uniquely regulates immunoglobulin gene class switch recombination (CSR) in activated B cells and their differentiation into plasma cells (Sciammas et al. 2006). Conversely, IRF8 has a dominant function in regulating the proliferation of myeloid progenitors and their differentiation into macrophages (Tamura et al. 2000).

The observations that IRF4 regulates differentiation of $\mathrm{T}$ cells into effector states such as Th2 and Th17 raised a molecular conundrum because the aforementioned Thelper cells express very low levels of the interaction partner PU.1 or the related Ets factor Spi-B (Lohoff et al. 2002). These findings suggested the existence of a novel partner for IRF4 and IRF8. ChIP-seq analysis of IRF4-targeted sequences in T cells revealed that IRF4 binds to a novel set of AP-1-IRF composite elements (AICE) (Ciofani et al. 2012; Glasmacher et al. 2012; Li et al. 2012; Tussiwand et al. 2012). Strikingly, this involves cooperative assembly of IRF4 with AP-1 heterodimers containing a basic leucine zipper transcription factor, AFT-like (BATF) subunit. This molecular property is shared by IRF 8 but not by other IRF family members, reminiscent of the molecular specificity of PU.1-IRF4 or PU.1-IRF8 complexes (Glasmacher et al. 2012). Thus, IRF4 and IRF8 proteins have evolved not only to interact with specific members of the Ets superfamily, namely PU.1 and Spi-B, but also with particular members of the AP-1 superfamily, namely, BATF-containing heterodimers. Intriguingly, these select members of the Ets and AP-1 family also have their expression largely confined to cells of the immune system. Therefore, considerable protein evolution within the IRF, Ets, and AP-1 families has enabled the establishment of unique immune-specific partnerships. In this perspective, major biological functions of IRF4 and IRF8 within the immune system are discussed in light of these two types of immunespecific transcription factor complexes.

\section{ROLES OF IRF4 AND IRF8 IN B-CELL DEVELOPMENT AND ACTIVATION}

IRF4 was cloned using a cDNA expression library and a DNA probe that corresponded to an EICE present in an immunoglobulin (Ig) $\lambda$ light-chain gene enhancer (Eisenbeis et al. 1995). As anticipated by earlier biochemical analysis, the recombinant protein was shown to cooperatively bind to EICE sequences present in $\operatorname{Ig} \lambda$ as well as Ig $\kappa$ gene enhancers. Based on these biochemical properties, the protein was initially termed Pip for PU.1 interaction partner and later redesignated as IRF4 based on its

\footnotetext{
${ }^{2}$ Current address: Division of Immunobiology and the Center for Systems Immunology, Cincinnati Children's Hospital Medical Center, Cincinnati, OH 45229. ${ }^{3}$ Current address: Helmholtz Zentrum München, 85764 Neuherberg, Germany 
relatedness to other IRF family members, particularly IRF8. cDNA clones of IRF4 (LSIRF/ICSAT) were also isolated by two other laboratories based on its expression in lymphocytes (Matsuyama et al. 1995; Yamagata et al. 1996). The binding of IRF4, in a cooperative manner with the transcription factor PU.1, to functionally important regulatory motifs in the $\operatorname{Ig} \kappa 3^{\prime}$ and $\lambda$ enhancers suggested an important role in the developmental activation of $\mathrm{Ig}$ light-chain transcription and rearrangement. Analysis of IRF4 knockout (KO) mice did not reveal a defect in B-cell development. However, the combined loss of IRF4 and IRF8 resulted in a profound block to B-cell development, precisely at the cycling pre-B-cell stage (Lu et al. 2003). The $\operatorname{Irf} 4^{-1-}, \operatorname{Irf8^{-/-}}$ pre-B cells were impaired for the activation of germline $\kappa$ and $\lambda$ gene transcription as well as DNA recombination. Thus, the genetic analysis satisfied the molecular predictions and suggested that IRF4 and IRF8 were molecularly redundant in the context of pre-B cells. This was formally showed by complementing $\operatorname{Irf4} 4^{-1-}, \operatorname{Irf} 8^{-/-}$pre-B cells with IRF4 or IRF8 (Ma et al. 2006).

Although IRF4 is not required for B-cell development, it regulates B-cell activation and has been shown to orchestrate Ig CSR as well as plasma cell differentiation (Sciammas et al. 2006). Even though mature B cells express both IRF4 and IRF8, in this cellular context the two transcription factors do not function in a molecularly redundant or equivalent manner. IRF4 promotes CSR in activated B cells and their differentiation into plasma cells by positively regulating the expression of the Aicda and Blimp1 genes, respectively. Importantly, these genes are reflective of two mutually antagonistic programs of B-cell gene expression. IRF4 controls the transition between the competing programs of gene expression in a concentration-dependent manner with low levels favoring CSR and higher levels inducing plasma cell differentiation. Based on these findings, IRF4 was also proposed to regulate the mutually antagonistic programs of gene expression that are characteristic of germinal center B cells and plasma cells. Furthermore, it was suggested to function as a key component of a gene regulatory network that enables B cells upon their activation to transiently acquire the GC state before collapsing it and undergoing terminal differentiation into plasma cells.

Recently, using both a constitutive and a conditional knockout of Irf4, it has been established that IRF4 is required for the generation of germinal center B cells (Ochiai et al. 2013). IRF4 controls the expression of the $B c l 6$ and $O b f 1$ genes, both of which are required for the germinal center response. A tet-inducible allele encoding IRF4 has been used to incisively test the concentrationdependent functioning of IRF4 in B-cell-fate dynamics. Transient induction of IRF4 enables the generation of germinal center B cells but not plasma cells. Under these conditions, the $\operatorname{Irf} 4^{-/}$B cells carrying the inducible Irf4 allele are not able to sustain a high concentration of IRF4. Conversely sustained and high expression levels of IRF4 favor plasma cell generation at the expense of GC B cells. How do differing concentrations of IRF4 in activated $B$ cells result in activation as well as repression of distinct sets of genes? ChIP-seq experiments with B cells activated in vitro have provided an important molecular clue. During the initial stages of B-cell activation, IRF4 is seen to bind predominantly to EICE motifs. At a later time point when plasma cells expressing higher levels of IRF4 are generated, there is increased occupancy of IRF4 at ISRE motifs. These latter motifs represent interferonstimulated response elements that are bound with low affinity by IRF4 homodimers. Intriguingly, these IRF4bound ISRE motifs are enriched in genes that are preferentially expressed in plasma cells. These results have led to the proposal that plasma cell differentiation is dependent on sustained and higher levels of IRF4 expression because this enables efficient occupancy of low-affinity ISRE motifs that are associated with plasma cell genes.

\section{DISCOVERY OF IRF4-BATF COMPLEXES REGULATING HELPER-T-CELL DIFFERENTIATION}

IRF4 is important for the differentiation of various Thelper (Th)-cell subsets, including Th2, Th9, Th17, and Tfh cells (Glasmacher et al. 2012). The role for IRF8 in Th differentiation has not been as intensively investigated. Interestingly, IRF8 KO T cells more readily differentiate into Th17 cells, in contrast with their wild-type counterparts (Ouyang et al. 2011). Thus, IRF8 appears to antagonize the function of IRF4 in this cellular context.

Until recently, the molecular mechanisms by which IRF4 and IRF8 might regulate gene expression in helper $\mathrm{T}$ cells had remained enigmatic. It had been suggested that in Th2 cells, IRF4 could interact with nuclear factor of activated T cells (NFAT) to regulate interleukin-4 expression (Rengarajan et al. 2002), whereas in T cells that respond to IL-21, IRF4 was proposed to interact with STAT3 to regulate Blimp1 expression (Kwon et al. 2009). However, the generality of these interactions and the biochemical mechanisms underlying DNA assembly remained to be elucidated. As mentioned previously, IRF4 and IRF8, unlike other members of the IRF factor family, bind with low affinity to ISREs but can be recruited to high-affinity EICE motifs in B cells by interacting with the Ets family transcription factors, PU.1 and Spi-B. However, PU.1 and Spi-B are expressed at very low levels in T-helper-cell lineages, except in Th9 cells. Recently, four independent studies have described a novel molecular partnership by which IRF4 and IRF8 regulate gene expression in Th cells (Ciofani et al. 2012; Glasmacher et al. 2012; Li et al. 2012; Tussiwand et al. 2012). Through ChIP-seq experiments, IRF4 and BATF were shown to cooperatively assemble on unusual DNA elements to regulate differentiation and functioning of Th17 cells. The cobound genomic target sequences contained AICE motifs. BATF is an immune-specific AP-1 family member that, similar to IRF4, is required for differentiation of Th2, Tfh, and Th17 cells as well as CSR in B cells (Murphy et al. 2013). In the context of Th17 cells, IRF4 and BATF cooperatively bind to the promoters and presumptive enhancer regions of the $I l 17 a, I l 21, I l 23 r$, and 
IL12 rb1 loci to regulate their activation. Importantly, the activation of these genes is also dependent on the transcription factors Stat3 and Roryt, thereby revealing a combinatorial set of regulators that program gene expression reflective of the Th17 effector state. IRF4 and BATF complexes could similarly function in a combinatorial manner with other transcription factors to regulate distinct Th effector states.

\section{ROLES OF IRF4 AND IRF8 IN PROGRAMMING DENDRITIC CELLS FOR ANTIGEN PRESENTATION}

IRF4 and IRF8 are differentially expressed within dendritic cell (DC) subsets (Tamura et al. 2005). IRF4 regulates development of $\mathrm{CD}_{11} \mathrm{~b}^{+}$DCs that appear to be specialized for antigen presentation to MHCII-restricted helper T cells. On the other hand, IRF8 controls development of $\mathrm{CD}_{11 \mathrm{~b}}{ }^{-} \mathrm{DC}$ populations that preferentially present antigens to MHCI-restricted cytotoxic T cells. The reciprocal expression of IRF4 and IRF8 in CD11 $b^{+}$ and $\mathrm{CD}_{11 \mathrm{~b}^{-}} \mathrm{DC}$ populations raised the possibility that the divergent expression of IRF4 and IRF8 could underlie the functionally specialized states of these DC populations (Vander Lugt et al. 2014). By using a floxed allele of IRF4 and conditionally deleting it in the DC compartment, it has been recently shown that IRF4 is required in DCs to enable efficient priming of Th responses in vivo as well as in vitro (Vander Lugt et al. 2014). IRF4 appears to do so by binding to and activating genes encoding components of the MHCII antigen processing and presentation pathway. The molecular basis by which IRF4 in contrast with IRF8 might selectively activate these genes remains to be elucidated.

As is the case with antigen processing and presentation, emerging evidence suggests that IRF4 and IRF8 diverge in their regulation of cytokine genes in DCs. IRF8 is a critical regulator of $I l 12 b$ in DCs and IRF4 cannot substitute for this function (Tamura et al. 2005). Accordingly, $I l 12 b$ transcripts are primarily detected in IRF8-dependent DC subsets. In contrast, IRF4-dependent DCs express higher levels of the $I l 23 a$ gene, which encodes the p19 subunit of the IL-23 cytokine. The differential expression of IL-23 and IL-12 components suggests that IRF4and IRF8-dependent DCs might make distinct contributions to priming Th17 versus Th1 differentiation, respectively. In line with this possibility, IRF4-dependent DCs have a critical role in priming Th17 responses. Similarly, Th2 responses are essentially undetectable in mice lacking IRF4-dependent DCs, indicating that IRF4 also has a particularly critical role in programming DCs to initiate Type II responses (Williams et al. 2014). Thus, IRF4 functions in a cell-intrinsic manner to regulate both the DC and the Th-cell compartment in the context of adaptive Th2 and Th17 immune responses.

Analysis of sequences targeted by IRF4 and IRF8 in DCs reveals predominant use of the EICE motif, suggesting that the primary mode of recruitment of IRF4 and IRF8 to regulatory elements in DCs is via cooperative assembly with PU.1 or the related Ets factor Spi-B (Glasmacher et al. 2012). However, current evidence also supports a key role of IRF4 or IRF8 complexes with BATFcontaining AP-1 heterodimers in DCs. Whereas IRF4targeted sequences in DCs show strongest enrichment for the EICE motif, $\sim 15 \%$ contain an AICE motif. Furthermore, BATF3 expression is highly correlated with IRF8 expression among DC subsets, and loss of either BATF3 or IRF8 results in the selective loss of BATF3 and IRF8 coexpressing subsets (Murphy et al. 2013). Although complementation of BATF3-deficient progenitors with BATF1 or BATF2 is sufficient to rescue development of these DC subsets, indicating functional redundancy among BATF family members, mutant variants of BATF that retain DNA-binding activity but that cannot cooperatively assemble with IRF4 and IRF8 are functionally defective (Glasmacher et al. 2012; Tussiwand et al. 2012). It remains to be determined whether development of IRF4-dependent DC subsets is similarly dependent on interactions between IRF4 and BATF family members.

\section{IRF4 AND IRF8: EVOLVING NEW MODES OF DNA ASSEMBLY}

PU.1 binding to the Ets site of the EICE motif (GGAANNGAAA) facilitates the binding of IRF4 to the adjacent IRF site (GGAANNGAAA). Importantly, IRF8 can substitute for IRF4 and cooperatively bind with PU.1. However, alternative IRF members, IRF1 and IRF2, that bind with high affinity to the IRF site within the EICE motif, fail to cooperatively assemble with PU.1 (Brass et al. 1999). In fact, the DNA-binding domains (DBD) of IRF1 and IRF2 show anticooperative interactions with PU.1 and can displace PU.1 from the composite sites. Similarly, Spi-B can substitute for PU.1 and recruit IRF4 or IRF8 to the EICE composite elements. Structural studies of the DBDs of other Ets family members, $\operatorname{GABP} \alpha$, SAP-1, and Ets1 suggest that these factors should be unable to interact with IRF4 and IRF8.

As noted above, IRF4 and IRF8 also use BATF-containing AP-1 complexes as binding partners. Biochemical studies have shown that a JunB-BATF but not a JunBFosL2 heterodimer is able to recruit IRF4 or IRF8 to AICE motifs (Glasmacher et al. 2012). Thus, as with the case of the observed specificity among Ets and IRF family members, selectivity is similarly observed among AP-1 family members for complex formation with IRF4 and IRF8.

Unlike the EICE motif, the AICE motif occurs in two configurations: an IRF site abutting an AP-1 site (GAAA TGAGTCA, Bc11b gene) or an inverted IRF site separated by 4 bp from the AP-1 site (TTTCNNNNTGACTAA, CTLA-4 gene) (Glasmacher et al. 2012). Biochemical experiments have shown that JunB-BATF and IRF4 can cooperatively assemble on both types of AICE motifs. Interestingly, IRF4 binding to DNA sequences that differ in the orientation or spacing of the IRF site is dramatically reduced, thereby demonstrating a stereo-specific requirement for assembly of the complexes. Although structural 
models of IRF4-BATF-containing complexes bound to the two distinct types of AICE motifs have been proposed (Glasmacher et al. 2012), atomic structures of these complexes will be needed to account for this surprising and enigmatic property.

\section{CONCLUSIONS}

IRF4 and IRF8 are immune-specific members of the IRF transcription factor family that have evolved to perform major functions in regulating the development and differentiation of innate as well as adaptive cells. These molecular functions rely on novel molecular complexes with specific members of the Ets and AP-1 families. We propose that such distinctive and highly specific transcription factor partnerships are most likely the result of the emergence of new genomic regulatory DNA elements containing EICE or AICE motifs that control the activity of a diverse array of immune response genes. In this scenario, selected members of the IRF, Ets, and AP-1 families have undergone considerable protein evolution to enable their specific interactions with one another and thereby cooperative assembly on the unusual composite DNA elements. In this fashion, the functions of other IRF, Ets, and AP-1 transcription factors in other cell types including immune cells would have remained unperturbed. Thus, the emergence of immune-specific genomic regulatory elements as well as of new members within the IRF, Ets, and AP-1 super families that can cooperatively assemble on the composite sites to regulate unique patterns of gene activity is likely to provide new evolutionary insights into the mammalian immune system.

\section{REFERENCES}

Brass AL, Zhu AQ, Singh H. 1999. Assembly requirements of PU.1-Pip (IRF-4) activator complexes: Inhibiting function in vivo using fused dimers. $E M B O J$ 18: 977-991.

Ciofani M, Madar A, Galan C, Sellars M, Mace K, Pauli F, Agarwal A, Huang W, Parkurst CN, Muratet M, et al. 2012. A validated regulatory network for Th17 cell specification. Cell 151: 289-303.

Driggers PH, Ennist DL, Gleason SL, Mak WH, Marks MS, Levi BZ, Flanagan JR, Appella E, Ozato K. 1990. An interferon gamma-regulated protein that binds the interferoninducible enhancer element of major histocompatibility complex class I genes. Proc Natl Acad Sci 87: 3743-3747.

Eisenbeis CF, Singh H, Storb U. 1995. Pip, a novel IRF family member, is a lymphoid-specific, PU.1-dependent transcriptional activator. Genes Dev 9: 1377-1387.

Glasmacher E, Agrawal S, Chang AB, Murphy TL, Zeng W, Vander Lugt B, Khan AA, Ciofani M, Spooner CJ, Rutz S, et al. 2012. A genomic regulatory element that directs assembly and function of immune-specific AP-1-IRF complexes. Science 338: $975-980$.

Kwon H, Thierry-Mieg D, Thierry-Mieg J, Kim HP, Oh J, Tunyaplin C, Carotta S, Donovan CE, Goldman ML, Tailor $\mathrm{P}$, et al. 2009. Analysis of interleukin-21-induced Prdm1 gene regulation reveals functional cooperation of STAT3 and IRF4 transcription factors. Immunity 31: 941-952.
Li P, Spolski R, Liao W, Wang L, Murphy TL, Murphy KM, Leonard WJ. 2012. BATF-JUN is critical for IRF4-mediated transcription in T cells. Nature 490: 543-546.

Lohoff M, Mittrücker HW, Prechtl S, Bischof S, Sommer F, Kock S, Ferrick DA, Duncan GS, Gessner A, Mak TW. 2002. Dysregulated $T$ helper cell differentiation in the absence of interferon regulatory factor 4. Proc Natl Acad Sci 99: $11808-11812$.

Lu R, Medina KL, Lancki DW, Singh H. 2003. IRF-4,8 orchestrate the pre-B-to-B transition in lymphocyte development. Genes Dev 17: 1703-1708.

Ma S, Turetsky A, Trinh L, Lu R. 2006. IFN regulatory factor 4 and 8 promote Ig light chain kappa locus activation in pre-B cell development. J Immunol 177: 7898-7904.

Matsuyama T, Grossman A, Mittrücker HW, Siderovski DP, Kiefer F, Kawakami T, Richardson CD, Taniguchi T, Yoshinaga SK, Mak TW. 1995. Molecular cloning of LSIRF, a lymphoid-specific member of the interferon regulatory factor family that binds the interferon-stimulated response element (ISRE). Nucleic Acids Res 23: 2127-2136.

Murphy TL, Tussiwand R, Murphy KM. 2013. Specificity through cooperation: BATF-IRF interactions control immune-regulatory networks. Nat Rev Immunol 13: 499509.

Ochiai K, Maienschein-Cline M, Simonetti G, Chen J, Rosenthal R, Brink R, Chong AS, Klein U, Dinner AR, Singh H, et al. 2013. Transcriptional regulation of germinal center B and plasma cell fates by dynamical control of IRF4. Immunity 38: $918-929$.

Ouyang X, Zhang R, Yang J, Li Q, Qin L, Zhu C, Liu J, Ning H, Shin MS, Gupta M, et al. 2011. Transcription factor IRF8 directs a silencing programme for TH17 cell differentiation. Nat Commun 2: 314.

Rengarajan J, Mowen KA, McBride KD, Smith ED, Singh H, Glimcher LH. 2002. Interferon regulatory factor 4 (IRF4) interacts with NFATc2 to modulate interleukin 4 gene expression. J Exp Med 195: 1003-1012.

Sciammas R, Shaffer AL, Schatz JH, Zhao H, Staudt LM, Singh H. 2006. Graded expression of interferon regulatory factor-4 coordinates isotype switching with plasma cell differentiation. Immunity 25: 225-236.

Tamura T, Nagamura-Inoue T, Shmeltzer Z, Kuwata T, Ozato K. 2000. ICSBP directs bipotential myeloid progenitor cells to differentiate into mature macrophages. Immunity 13: 155165.

Tamura T, Tailor P, Yamaoka K, Kong HJ, Tsujimura H, O'Shea JJ, Singh H, Ozato K. 2005. IFN regulatory factor-4 and -8 govern dendritic cell subset development and their functional diversity. J Immunol 174: 2573-2581.

Tussiwand R, Lee WL, Murphy TL, Mashayekhi M, Wumesh KC, Albring JC, Satpathy AT, Rotondo JA, Edelson BT, Kretzer NM, et al. 2012. Compensatory dendritic cell development mediated by BATF-IRF interactions. Nature 490: 502-507.

Vander Lugt B, Khan AA, Hackney JA, Agrawal S, Lesch J, Zhou M, Lee WP, Park S, Xu M, DeVoss J, et al. 2014. Transcriptional programming of dendritic cells for enhanced MHC class II antigen presentation. Nat Immunol 15: $161-$ 167.

Williams JW, Tjota MY, Clay BS, Vander Lugt B, Bandukwala HS, Hrusch CL, Decker DC, Blaine KM, Fixsen BR, Singh H, et al. 2014. Transcription factor IRF4 drives dendritic cells to promote Th2 differentiation. Nat Commun 4: 2990.

Yamagata T, Nishida J, Tanaka S, Sakai R, Mitani K, Yoshida M, Taniguchi T, Yazaki Y, Hirai H. 1996. A novel interferon regulatory factor family transcription factor, ICSAT/Pip/ LSIRF, that negatively regulates the activity of interferonregulated genes. Mol Cell Biol 16: 1283-1294. 


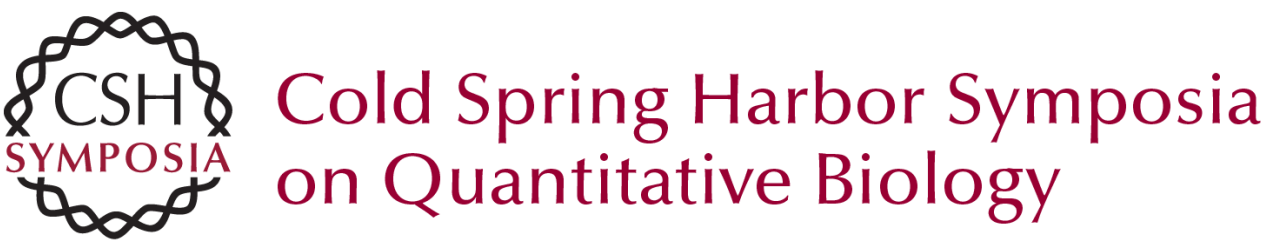

\section{The Molecular Choreography of IRF4 and IRF8 with Immune System Partners}

Harinder Singh, Elke Glasmacher, Abraham B. Chang, et al.

Cold Spring Harb Symp Quant Biol 2013 78: 101-104 originally published online April 21, 2014 Access the most recent version at doi:10.1101/sqb.2013.78.020305

References This article cites 22 articles, 10 of which can be accessed free at: http://symposium.cshlp.org/content/78/101.full.html\#ref-list-1

License

Email Alerting Receive free email alerts when new articles cite this article - sign up in Service the box at the top right corner of the article or click here. 